\title{
Influences Des Modes D'accès A La Terre Sur La Production Agricole Dans Les Communes De Dassa-Zoumé Et De Glazouè Au Centre Du Bènin
}

\author{
Kadjegbin Toundé Roméo Gislain \\ Laboratoire d'Etudes des Dynamiques Urbaines et Régionales (LEDUR), \\ Département de Géographie et Aménagement du Territoire (DGAT), \\ Université d'Abomey-Calavi (UAC, République du Bénin) \\ Yabi Ibouraima \\ Adjakpa T. Théodore \\ Kotchare Parfaite \\ Sewade Sokegbe Grégoire \\ Houssou Christophe Sègbè
}

Laboratoire Pierre Pagney 'Climat, Eau, Ecosystème et Développement'

(LACEEDE), Département de Géographie et Aménagement du Territoire

(DGAT), Université d'Abomey-Calavi (UAC, République du Bénin)

Doi: 10.19044/esj.2018.v14n6p412 URL:http://dx.doi.org/10.19044/esj.2018.v14n6p412

\begin{abstract}
The land plays an important role in the social and economic life of the populations of all of the world in general and in the rural world in particular in such a way that it constitutes the essential support of every agricultural activity. The purpose of this article is to study the influence of the access to land on the agricultural production in the communes of Dassa-Zoumé and Glazoué. From a sample of 279 farm households, the study of the influence of land access on the agricultural production in the communes of Dassa-Zoumé and Glazoué was made using the questionnaire sent to farmers and CARDER/Zou authorities. In order to collect reliable information, a presurvey was carried out at first, followed by an individual interview, a focus group and a MARP ( Active Participatory Research Method). Similarly, the SWOT analyses model was used and the Ruthenberg coefficient was calculated to better appreciate the cropping system used in both communities. At the end of the analyses, we can notice that in the communes of DassaZoumé and Glazoué, inheritance remains the main modes of access to land with $82,9 \%$, followed by "donation" with $5,1 \%$, then the borrowingwith $4,3 \%$ and the collective appropriation(2,6\%), renting $(1,7 \%)$, sharecropping $(1,7 \%)$ and the purchase $(1,7 \%)$. OF these different modes of access, the inheritance
\end{abstract}


is the most privileged while renting, sharecropping and buying constitute binding modes. These different modes of access determine the different uses that can be made of rural land and constitute either a constraint or an asset for agricultural production.

Keywords: Communes of Dassa-Zoumé and Glazoué ( center of Bénin), modes of access to land, agricultural production, food safety

\section{Résumé}

La terre joue un rôle très important dans la vie sociale et économique des populations de tous les pays du monde en général et dans le monde rural en particulier en ce sens qu'elle constitue le support incontournable de toute activité agricole. L'objectif de cet article est d'analyser l'influence des modes d'accès à la terre sur la production agricole dans les Communes de DassaZoumé et de Glazoué. A partir d'un échantillonnage composé de 279 ménages agricoles, l'étude de l'influence des modes d'accès à la terre sur la production agricole dans les Communes de Dassa-Zoumé et de Glazoué a été faite à l'aide du questionnaire adressé aux exploitants agricoles et aux responsables du CARDER/Zou. Dans l'optique de collecter des informations fiables, une préenquête a été réalisée au prime abord suivi d'un entretien individuel, d'un focus group et d'une MARP (Méthode Active de Recherche Participative). De même, le modèle d'analyse SWOT a été utilisé et le coefficient de Ruthenberg calculé pour mieux apprécier le système de culture pratiqué dans les deux (02) communes. Au terme des analyses, il est constaté que dans les Communes de Dassa-Zoumé et de Glazoué, l'héritage demeure le principal mode d'accès à la terre avec $82,9 \%$ suivi du ' don' ' avec 5,1\%, de l'emprunt avec $4,3 \%$ et de l'appropriation collective $(2,6 \%)$, du fermage ou location $(1,7 \%)$, le métayage $(1,7 \%)$ et l'achat $(1,7 \%)$. De ces différents modes d'accès, l'héritage est le plus privilégié tandis que la location, le métayage et l'achat constituent des modes contraignants. Ces différents modes d'accès déterminent les différents usages qui peuvent être faits de la terre rurale et constituent soit une contrainte ou un atout pour la production agricole.

Mots-clés : Communes de Dassa-Zoumè et de Galzoué (Centre du Bénin), modes d'accès à la terre, production agricole, sécurité alimentaire

\section{Introduction}

L'agriculture est une activité rurale qui a pour vocation première de nourrir la population d'une localité, d'un pays. Elle fait appel, entre autres, à la terre en tant que support des cultures à développer (Boko, 2004).En effet, l'accès à la terre est depuis des temps immémoriaux une question vitale pour toute société. Les groupes sociaux ont élaboré des règles écrites ou non pour préciser 
l'usage, le partage et la transmission de ce bien précieux entre tous. Car, ce bien "'sacré', ( ) est en même temps l'objet de toutes les contestations et conflits ; ce caractère sacré est particulièrement marqué en Afrique subsaharienne. La quasi-totalité des auteurs ayant rédigés des articles ou des ouvrages relatifs au foncier y font référence (Erpicum, 2004).

En Afrique, la moitié des terres cultivées est affectée par la dégradation et l'érosion des sols. De même, jusqu'à $80 \%$ des pâturages donne des signes de détérioration (Houédjissin, 2000). Ainsi, les pays en développement surtout ceux d'Afrique au sud du Sahara connaissent depuis quelques années une dégradation de leur situation alimentaire. Par ailleurs, il est évident de constater une diminution globale de l'espace agricole qui s'explique par la pression foncière opérée lors des divers lotissements (Djaouga, 2006). Entre 1976 et 1997, environ $46 \%$ des Béninois ont connu une pénurie alimentaire suite aux sécheresses (Afouda, 1990 ; Odjo, 1997 ; Boko, 1998).Les exploitants agricoles se plaignent de la "fatigue" de leurs sols parce qu'ils constatent que les rendements agricoles baissent considérablement malgré des investissements en temps de travail de plus en plus élevés (Agossou et Igué, 2002).

Face à cette situation, le besoin d'améliorer la productivité de la terre et du travail apparaît comme une priorité. A ces besoins s'ajoute la nécessité de préserver les ressources naturelles et la biodiversité par la promotion des modes d'exploitations durables et reproductibles.

A l'instar des autres localités rurales du Bénin, la terre joue un rôle très important dans la vie sociale et économique des populations des Communes de Dassa-Zoumè et de Glazoué en ce sens qu'elle constitue le support incontournable de toute activité agricole. Le statut social de l'individu ou du groupe familial dont il est issu conditionne son acquisition.

Quels sont les modes d'accès à la terre dans le secteur d'étude ?

Quelles sont les influences de ces modes d'accès sur les ménages agricoles ?

\section{1-Matériels et méthodes}

\subsection{Cadrage géographique de l'étude}

Le secteur d'étude porte sur les Communes de Dassa-Zoumè et de Glazoué (figure 1). Il est situé entre $1^{\circ} 41^{\prime}$ et $2^{\circ} 39^{\prime}$ de longitude est et $7^{\circ} 27^{\prime}$ et $8^{\circ} 31^{\prime}$ de latitude Nord. Le secteur d'étude est limité au nord par la Commune de Bassila dans le département de la Donga, au Sud par les Communes de Djidja, de Covè et de Zagnanado dans le département du Zou, à l'Est par les Communes de Savè et Ouèssè, et à l'Ouest par les Communes de Bantè et de Savalou. Il couvre une superficie de $3461 \mathrm{~km} 2$ et une population de 197817 habitants (INSAE, 2013). 


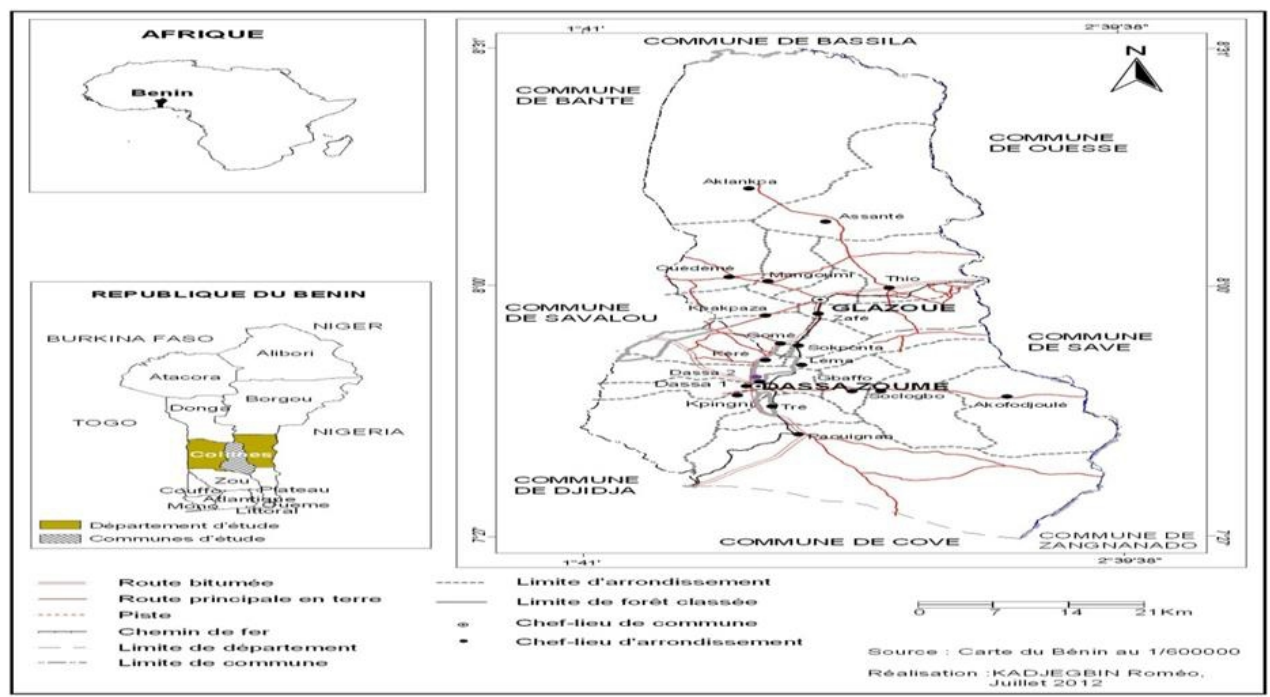

Figure 1 : Situations géographiques et subdivisions administratives des Communes de Dassa-Zoumè et de Glazoué

La collecte des informations sur le terrain a nécessité une approche méthodologique appropriée.

\subsection{Nature des données collectées lors des enquêtes de terrain}

\subsubsection{Outils et techniques de collecte des données}

Dans le cadre des enquêtes de terrain, deux (02) outils d'investigation ont été utilisés. Il s'agit desquestionnaires et d'une grille d'observation.

- Le questionnaire est élaboré suivant les objectifs spécifiques. Il a permis de collecter des informations auprès des ménages agricoles.

- La grille d'observation quant à elle, a servi à faire des observationssur les systèmes culturaux utilisés par les exploitants agricoles.

Dans le souci de recueillir le maximum d'informations et de données fiables, plusieurs techniquesontété adoptées. La Méthode Active de Recherche Participative (MARP) qui s'intéresse aux réalités quotidiennesdes acteurs concernés par l'enquête à rendre possible les investigations dans le secteur d'étude. La méthode de focus-group a pris en compte les femmes productrices puisqu'elles constituent la couche cible d'enquête et a également contribué à confronter et à compléter les informations collectées à l'aide des questionnaires. 


\subsection{2- Echantillonnage}

\section{Critères de choix des personnes enquêtées}

Le choix des personnes enquêtées a été fait suivant la méthode de choix raisonné. Cette méthode est utilisée pour déterminer l'effectif de la population agricole enquêtée de même que le nombre de champs visités.

Deux (02) critères ont déterminé le choix des personnes enquêtées:

- être exploitant agricole ;

- avoir une expérience d'au moins 10 ans.

Dans l'optique d'aboutirà des résultats significatifs, seuls les arrondissements à forte potentialité agricole sont choisis.

Pour déterminer la taille de l'échantillon, la formule de Schwarz (1995) est utilisée. Ainsi, si n désigne la taille de l'échantillon, on a :

$$
\mathrm{n}=\mathrm{Z} \alpha^{2} \times \mathrm{pq} / \mathrm{i}^{2}
$$

$\mathrm{Z} \alpha=$ écart réduit correspondant à un risque $\alpha$ de $5 \%$

$\mathrm{p}=$ proportion des ménages agricoles par rapport au nombre de ménages dans les Communes de Dassa-Zoumè et de Glazoué (effectif de ménages agricoles en $2013=25538$; effectif total de ménages en $2013=33532$ ) soit $\mathrm{p}=76,16 \%$ $\mathrm{i}=$ précision désirée égale à $5 \%$

$\mathrm{q}=1-\mathrm{p}($ ici, $\mathrm{q}=23,84 \%)$

$\mathrm{n}=(1,96)^{2} \times 0,7616(1-0,7616) / 0,05^{2}=279$ ménages

Les 279 ménages enquêtés ont été répartis dans les arrondissements dont le nombre varie d'une commune à une autre en fonction de la production agricole. Le choix du nombre de ménages par arrondissement s'est effectué suivant la règle de proportionnalité, ce qui a permis d'obtenir la répartition du tableau I.

Tableau I : Répartition spatiale de la population enquêtée et le nombre de champs visités

\begin{tabular}{|c|c|c|c|c|c|}
\hline Communes & Arrondissements & $\begin{array}{c}\text { Effectif total } \\
\text { des ménages } \\
\text { agricoles }\end{array}$ & $\begin{array}{c}\text { Nombre de } \\
\text { ménages } \\
\text { enquêtés }\end{array}$ & $\begin{array}{c}\text { Pourcentage } \\
\mathbf{( \% )}\end{array}$ & $\begin{array}{c}\text { Nombre de } \\
\text { champs } \\
\text { visités }\end{array}$ \\
\hline \multirow{3}{*}{$\begin{array}{c}\text { Dassa- } \\
\text { Zoumè }\end{array}$} & PAOUINGNAN & 4145 & 66 & 1,58 & 15 \\
\cline { 2 - 6 } & SOCLOGBO & 2062 & 33 & 1,58 & 7 \\
\cline { 2 - 6 } & KERE & 1247 & 20 & 1,58 & 4 \\
\cline { 2 - 6 } & DASSA II & 1236 & 19 & 1,54 & 4 \\
\cline { 2 - 6 } & KPINGNI & 1004 & 16 & 1,58 & 4 \\
\hline \multirow{4}{*}{ Glazoué } & AKLANKPA & 2487 & 39 & 1,58 & 9 \\
\cline { 2 - 6 } & THIO & 1634 & 26 & 1,58 & 6 \\
\cline { 2 - 6 } & OUEDEME & 1318 & 21 & 1,58 & 5 \\
\cline { 2 - 6 } & ZAFFE & 1252 & 20 & 1,58 & 5 \\
\cline { 2 - 6 } & MAGOUMI & 1228 & 19 & 1,58 & 4 \\
\hline TOTAL & $\mathbf{1 0}$ & $\mathbf{1 7 6 1 3}$ & $\mathbf{2 7 9}$ & $\mathbf{1 , 5 8}$ & $\mathbf{6 3}$ \\
\hline
\end{tabular}

Source : Résultats destravaux de terrain, 2016 
Les critères du choix des champs visités sont fonction de leur importance relative (grande et petite superficie emblavée), de leur nature géographique (sols ferrugineux, sols hydromorphes, vertisols, ...) ainsi que des systèmes de culture mis en œuvre.

\subsection{3- Analyse des données}

Pour mieux apprécier le système de culture pratiqué dans les deux (02) communes, le coefficient de Ruthenberg noté $\mathbf{R}$ a été calculé.

$$
R=\frac{\mathrm{NC}}{N C+D J} \times 100
$$

$\mathrm{R}=$ Coefficient de Ruthenberg

$\mathrm{NC}=$ Nombre d'années de culture

$\mathrm{DJ}=$ Durée de la Jachère

On a donc les cas de figure suivants :

- si R > 66, on parlera d'un système de culture permanente ;

- si $\mathrm{R}<33$, on parlera d'un système de culture itinérante ;

- si $33<\mathrm{R}<66$, on parlera d'un système de jachère.

Ce coefficient $\mathrm{R}$ permet dans un premier temps d'identifier le type de système cultural dominant dans une localité donnée (Tenté et Sinsin, 2002 ; Wokou, 2009 ; Fangnon, 2012).

\section{2-Résultats}

\section{1-Modes d'accès à la terre dans les Communes de Dassa-Zoumé et de Glazoué}

L'accès à la terre, son acquisition et son utilisation répondent à des règles dont l'évolution dépend de chaque groupe socioculturel. Les différents modes d'accès à la terre enregistrés dans les Communes de Dassa-Zoumè et de Glazoué sont : l'héritage, l'achat, l'emprunt, le métayage, le fermage (location), le "don' et l'appropriation collective.

\subsection{1- Héritage}

Selon Biaou (1991) cité par Fangnon (2012), l'héritage foncier est un transfert de génération en génération des terres et de leurs ressources. Dans les Communes de Dassa-Zoumè et de Glazoué, l'héritage foncier autrefois patrilinéaire est de nos jours remise en cause. En effet, les enfants des deux (02) sexes (masculin et féminin) commencent à avoir droit à l'héritage même si chez la femme, cet acte d'héritage est toujours violemment critiqué. La pression démographique fait que la part de terrain revenant à chaque enfant s'amenuise considérablement. Dans ces conditions, naissent parfois des conflits fonciers.Les enquêtes de terrain ont attesté que 82,9 \% des exploitants agricoles (hommes et femmes) possèdent encore des terres héritées. Sur ces terres, les propriétaires sont en mesure de réaliser des cultures pérennes. La 
taille moyenne des domaines hérités est 6,2 ha à Dassa-Zoumè et 10,2 ha à Glazoué soit 8,2 ha pour l'ensemble des deux (02) communes avec des nuances intercommunales (tableau II).

Tableau II : Superficie moyenne des terres héritées et par sexe

\begin{tabular}{|c|c|c|c|c|}
\hline \multirow{3}{*}{ Communes } & Arrondissements & $\begin{array}{c}\text { Superficie moyenne des } \\
\text { terres héritées(ha) }\end{array}$ & $\begin{array}{c}\text { Part des } \\
\text { hommes (\%) }\end{array}$ & $\begin{array}{c}\text { Part des } \\
\text { femmes (\%) }\end{array}$ \\
\hline \multirow{4}{*}{$\begin{array}{c}\text { Dassa- } \\
\text { Zoumè }\end{array}$} & KERE & 06 & 66,7 & 33,3 \\
\cline { 2 - 5 } & KPINGNI & 02 & 66,7 & 33,3 \\
\cline { 2 - 5 } & PAOUINGNAN & 09 & 92,9 & 07,1 \\
\cline { 2 - 5 } & SOCLOGBO & 11 & 91,7 & 08,3 \\
\cline { 2 - 5 } & DASSA II & 03 & 50 & 50 \\
\hline \multirow{4}{*}{ Glazoué } & AKLAN-moyenne & $\mathbf{0 6 , 2}$ & 84,6 & $15, \mathbf{1}$ \\
\cline { 2 - 5 } & MAGOUMI & 15 & 100 & 00 \\
\cline { 2 - 5 } & OUEDEME & 07 & 85,7 & 14,3 \\
\cline { 2 - 5 } & THIO & 17 & 66,7 & 33,3 \\
\cline { 2 - 5 } & ZAFFE & 03 & $\mathbf{8 5 , 4}$ & $\mathbf{1 4 , 6}$ \\
\cline { 2 - 5 } & Sous-moyenne & 09 & $\mathbf{8 4 , 5}$ & $\mathbf{1 5 , 5}$ \\
\hline
\end{tabular}

Source :Enquête de terrain, juillet 2013

L'analyse du tableau II montre clairement que la femme est défavorisée dans le partage avec seulement $15,5 \%$ comme part moyenne d'héritage pour l'ensemble des deux (02) communes dont une prédominance de la Commune de Dassa-Zoumè (16,1\%) sur la Commune de Glazoué (14,6 $\%)$. En effet, destinée à une autre famille, la femme est généralement privée de l'héritage. Toutefois, cette tendance commence à être inversée, preuve que les droits des femmes commencent par être respectés dans les familles même si des poches de résistance persistent encore. Par ailleurs, la différence entre les superficies héritées dans les deux (02) communes s'explique par la densité de la population qui est plus forte à Dassa-Zoumè (55 hbts / km2 en 2013) qu'à Glazoué (52 hbts / km2 en 2013).

\subsection{2- Achat}

Selon Wartena (1987) cité par Fangnon (2012), le processus de "marchandisation" et de vente des terres a commencé depuis plus d'un siècle. Deux (02) catégories de vendeurs sont à noter à Dassa-Zoumè et à Glazoué :

- une première catégorie qui prend en compte ceux dont la terre héritée constitue un bien inaliénable et qui considèrent qu'elle ne saurait être vendue qu'en cas d'urgence (maladie, décès, etc.) ;

- une deuxième catégorie qui est constituée de ceux qui estiment que la terre héritée est vendue pour répondre aux exigences sociales (prise d'une deuxième ou troisième épouse, organisation de cérémonies funéraires, etc.). 
Deux (02) modes d'acquisition de la terre héritée peuvent s'observer à Dassa-Zoumè et à Glazoué :

- l'acquisition peut se faire par payement sur place monnayant l'obtention du titre foncier ;

- l'acquisition peut se faire également par payement en plusieurs tranches monnayant des conventions signées entre les parties contractantes, le délégué du village et des témoins.

Le coût moyen d'acquisition des terres varie de 20000 à 30000 FCFA le kanti $(600 \mathrm{~m} 2)$. Cette variation dépend de l'emplacement géographique du terrain par rapport aux agglomérations et de la qualité des sols qui s'y trouvent (fertilité par exemple). Les nouveaux acquéreurs sont souvent les fonctionnaires résidant en ville, les commerçants et les agences immobilières qui font la spéculation foncière. Les recherches de terrain montrent que 1,7 \% des agriculteurs enquêtés ont déclaré avoir acheté des terres agricoles. Sur les terres achetées, les propriétaires sont libres de faire toute sorte d'investissements sans crainte. Ce mode d'accès à la terre constitue dans certaines mesures des opportunités pour la production agricole. Le tableau III présente les superficies moyennes des terres achetées et les parts selon le sexe.

Tableau III: Superficie moyenne des terres achetées et par sexe

\begin{tabular}{|c|c|c|c|c|}
\hline \multirow{3}{*}{ Communes } & Arrondissements & $\begin{array}{c}\text { Superficie moyenne des } \\
\text { terres achetées (ha) }\end{array}$ & $\begin{array}{c}\text { Part des } \\
\text { hommes (\%) }\end{array}$ & $\begin{array}{c}\text { Part des } \\
\text { femmes } \\
(\mathbf{\%})\end{array}$ \\
\hline \multirow{4}{*}{$\begin{array}{c}\text { Dassa- } \\
\text { Zoumè }\end{array}$} & KERE & 0 & 0 & 0 \\
\cline { 2 - 5 } & KPINGNI & 0 & 0 & 0 \\
\cline { 2 - 5 } & PAOUINGNAN & 16 & 100 & 0 \\
\cline { 2 - 5 } & SOCLOGBO & 0 & 0 & 0 \\
\cline { 2 - 5 } & DASSA II & 0 & 0 & 0 \\
\hline \multirow{5}{*}{ Glazoué } & AKLAN-moyenne & $\mathbf{1 6}$ & 100 & 0 \\
\cline { 2 - 5 } & MAGOUMI & 20 & 0 & 0 \\
\cline { 2 - 5 } & OUEDEME & 0 & 0 & 0 \\
\cline { 2 - 5 } & THIO & 0 & 0 & 0 \\
\cline { 2 - 5 } & ZAFFE & 0 & $\mathbf{1 0 0}$ & $\mathbf{0}$ \\
\cline { 2 - 5 } & Sous-moyenne & $\mathbf{2 0}$ & $\mathbf{1 0 0}$ & $\mathbf{0}$ \\
\hline
\end{tabular}

Source : Enquêtes de terrain, juillet 2013

De l'analyse du tableau III, il ressort que les terres agricoles achetées dans les Communes de Dassa-Zoumè et de Glazoué se localisent uniquement dans les Arrondissements de Paouingnan et d'Aklankpa avec 16 ha en moyenne achetées à Paouingnanet 20 ha en moyenne achetées à Aklankpa. Dans les deux (02) communes, au nombre des acquéreurs figurentuniquement 
des hommes. Les femmes sont absentes du fait de leur situation économique précaire et de leur degré de pauvreté plus prononcé que celui des hommes. Cette situation n'est guère favorable à une production agricole satisfaisante puisque la production agricole pour son essor, a besoin d'une main d'œuvre abondante provenant des deux sexes (hommes et femmes). Or, si les femmes qui constituent après tout la majorité de la population se voient dans l'incapacité de s'acheter des terres du fait de leur situation économique précaire, cela constitue un frein pour la promotion du 'Genre' d'une part, et peut également amener cette frange importante de la population à abandonner l'agriculture et à se tourner vers d'autres secteurs d'activités plus rentables. Tout ceci compromet sérieusement la sécurité alimentaire dans le secteur d'étude.La satisfaction des besoins en terres agricoles amènent certains exploitants agricoles à contracter des prêts.

\subsection{3- Emprunt}

Dans les Communes de Dassa-Zoumè et de Glazoué, la terre est empruntée à qui en fait la demande auprès de son propriétaire presque gratuitement puisque l'emprunteur a le devoir d'entretenir l'espace emprunté ainsi que les cultures pérennes qui s'y trouvent.

Ce mode d'accès à la terre s'observe souvent entre les membres d'une même famille en l'occurrence l'épouse et son mari.Il est parfois étendu aux amis. Toutefois, ce mode d'accès à la terre ne sécurise pas l'emprunteur et constitue une contrainte pour la production agricolepuisque à tout moment le propriétaire peut réclamer son terrain même sans préavis ; ce qui expose l'emprunteur à tous les maux, d'où une inquiétude permanente de ce dernier.Il est à noter que la durée de l'emprunt n'est pas déterminée avec certitude et que l'emprunteur n'est pas autorisé à faire des cultures pérennes. Parmi les exploitants agricoles enquêtés, 04,3\% travaillent sur des terres empruntées. Le tableau IV montre les superficies moyennes des terres empruntées et les parts par sexe.

Tableau IV : Superficie moyenne des terres empruntées et par sexe

\begin{tabular}{|c|c|c|c|c|}
\hline Communes & Arrondissements & $\begin{array}{l}\text { Superficie moyenne des } \\
\text { terres empruntées (ha) }\end{array}$ & $\begin{array}{c}\text { Part des } \\
\text { hommes }(\%)\end{array}$ & $\begin{array}{c}\text { Part des } \\
\text { femmes } \\
(\%)\end{array}$ \\
\hline \multirow{6}{*}{ Dassa-Zoumè } & KERE & 1 & 0 & 100 \\
\hline & KPINGNI & 6 & 100 & 0 \\
\hline & PAOUINGNAN & 0 & 0 & 0 \\
\hline & SOCLOGBO & 0 & 0 & 0 \\
\hline & DASSA II & 0 & 0 & 0 \\
\hline & Sous-moyenne & 03,5 & 14,3 & 85,7 \\
\hline \multirow{3}{*}{ Glazoué } & AKLANKPA & 0 & 0 & 0 \\
\hline & MAGOUMI & 0 & 0 & 0 \\
\hline & OUEDEME & 0 & 0 & 0 \\
\hline
\end{tabular}




\begin{tabular}{|c|c|c|c|c|}
\hline & THIO & 3 & 100 & 0 \\
\cline { 2 - 5 } & ZAFFE & 0 & 0 & 0 \\
\cline { 2 - 5 } Sous-moyenne & $\mathbf{0 3}$ & $\mathbf{1 0 0}$ & 0 \\
\hline Total & $\mathbf{0 3 , 3}$ & $\mathbf{9 0}$ & $\mathbf{1 0}$ \\
\hline
\end{tabular}

Source : Enquêtes de terrain, juillet 2013

Le tableau IV montre que très peu de propriétaires terriensprêtent leur terrain. Etant donné que les femmes ont un faible pouvoir d'achat, elles empruntent plus les terres à cultiver que les hommes, ce qui se traduit par un fort pourcentage de $85,7 \%$ à Dassa-Zoumè et mais plus faible $(10 \%)$ à Glazoué. Ce faible taux de propriétaire qui cède leur terrain pour la production agricole constitue une contrainte pour la production agricole et compromet également la sécurité alimentaire et ceci plus à Galzoué qu'à Dassa-Zoumè puisque sans terre à cultiver, pas d'activité agricole.

L'assiette de l'emprunt étant exclusivement réservée aux familiers, et très peu étendue aux autres producteurs, ces derniers se voient contraints de trouver d'autres formules d'accès à la terre comme le métayage.

\subsection{4- Métayage}

Selon Wartena (1987) cité par Fangnon (2012),le métayage est un contrat d'exploitation agricole dans lequel le propriétaire donne à bail son domaine rural pour une durée déterminée contre partage des fruits et des pertes. Dans les Communes de Dassa-Zoumèet de Glazoué, le métayage qui a cours dans le milieu consiste à partager en trois parties les récoltes issues des cultures pratiquées. Le tiers de ce partage revient au propriétaire et les deux tiers aux métayers. C'est donc un système de partage qui est bénéfique pour toutes les parties contractantes. Ce système de partage appelé en langue Idatcha "ôya" est pratiqué pour remédier aux nombreux problèmes fonciers que subissent les populations. Selon les enquêtes de terrain, $100 \%$ des propriétaires ont affirmé avec fierté avoir droit aux cultures pérennes (anacardes, acajous, mangues, pommes, oranges, etc.). Il est noté que 1,7\% des exploitants agricoles enquêtés travaillent sur des terres mises en métayage. Le tableau Villustre les superficies moyennes des terres confiées aux métayers et les parts des hommes et des femmes.

Tableau V: Superficie moyenne des terres sous contrats de métayage et par sexe

\begin{tabular}{|c|c|c|c|c|}
\hline Communes & Arrondissements & $\begin{array}{c}\text { Superficie moyenne des } \\
\text { terres sous contrat de } \\
\text { métayage (ha) }\end{array}$ & $\begin{array}{c}\text { Part des } \\
\text { hommes (\%) }\end{array}$ & $\begin{array}{c}\text { Part des } \\
\text { femmes (\%) }\end{array}$ \\
\hline \multirow{4}{*}{$\begin{array}{c}\text { Dassa- } \\
\text { Zoumè }\end{array}$} & KERE & 01 & 0 & 100 \\
\cline { 2 - 5 } & KPINGNI & 0 & 0 & 0 \\
\cline { 2 - 5 } & PAOUINGNAN & 0 & 0 & 0 \\
\cline { 2 - 5 } & SOCLOGBO & 0 & 0 & 0 \\
\cline { 2 - 5 } & DASSA II & 0 & 0 & $\mathbf{1 0 0}$ \\
\cline { 2 - 5 }
\end{tabular}




\begin{tabular}{|c|c|c|c|c|}
\hline \multirow{4}{*}{ Glazoué } & AKLANKPA & 0 & 0 & 0 \\
\cline { 2 - 5 } & MAGOUMI & 0 & 0 & 0 \\
\cline { 2 - 5 } & OUEDEME & 0 & 0 & 0 \\
\cline { 2 - 5 } & THIO & 04 & 100 & 0 \\
\cline { 2 - 5 } & ZAFFE & 0 & 0 & 0 \\
\cline { 2 - 5 } & Sous-moyenne & $\mathbf{0 4}$ & $\mathbf{1 0 0}$ & $\mathbf{2 0}$ \\
\hline
\end{tabular}

Source : Enquêtes de terrain, juillet 2013

A travers l'analyse du tableau $\mathrm{V}$, il ressort que les superficies moyennes des terres du contrat de métayage sont faibles $(02,5$ ha) dans les Communes de Dassa-Zoumè et de Glazoué. On note toutefois une disparité au niveau des deux (02) communes. En effet, cette superficie moyenne des terres confiées à des métayers est plus élevée et exclusivement concentrée dans l'Arrondissement de Kéré (01 ha) à Dassa-Zoumè alors que dans la Commune de Glazoué, elle est concentrée exclusivement à Thio (04 ha). Seules les femmes ont recours à ce mode d'accès à Dassa-Zoumè alors qu'à Glazoué, on note le recours à ce mode d'accès par les deux sexes avec une forte propension chez les hommes $(80 \%)$.

La crise de confiance liée au fait que les métayers font de fausses déclarations en ce qui concerne la quantité de produits récoltés ou perdus qui prend de plus en plus des allures inquiétantes entre les propriétaires terriens et les métayers fait que le métayage est en voie de disparition dans les deux (02) communes et tend à être remplacé par la location des terres. Tout ceci constitue une contrainte pour la production agricole.

\subsection{5- Fermage}

Les enquêtes de terrain ont révélé que dans les Communes de DassaZoumè et de Glazoué, la durée contractuelle de la location de la terre est d'au plus quatre ans renouvelable une fois. La rente est payée selon la convenance du locataire. Elle peut être négociée en début du contrat, ou par an. Le coût moyen de location est de 50000 FCFA par an et par hectare. Il est noté que $01,5 \%$ des exploitants agricoles enquêtés travaillent sur des terres mises en location. Le tableau VI fait ressortir les superficies moyennes des terres louées et les proportions par sexe.

Tableau VI : Superficie moyenne des terres louées et par sexe

\begin{tabular}{|c|c|c|c|c|}
\hline Communes & Arrondissements & $\begin{array}{c}\text { Superficie moyenne } \\
\text { des terres louées (ha) }\end{array}$ & $\begin{array}{c}\text { Part des hommes } \\
(\boldsymbol{\%})\end{array}$ & $\begin{array}{c}\text { Part des } \\
\text { femmes (\%) }\end{array}$ \\
\hline \multirow{5}{*}{ Dassa-Zoumè } & KERE & 0 & 0 & 0 \\
\cline { 2 - 5 } & KPINGNI & 0 & 0 & 0 \\
\cline { 2 - 5 } & PAOUINGNAN & 0 & 0 & 0 \\
\cline { 2 - 5 } & SOCLOGBO & 0 & 0 & 0 \\
\cline { 2 - 5 } & DASSA II & 0 & 0 & 0 \\
\cline { 2 - 5 } & Sous-moyenne & 0 & 0 & 0 \\
\hline
\end{tabular}




\begin{tabular}{|c|c|c|c|c|}
\hline \multirow{4}{*}{ Glazoué } & AKLANKPA & 0 & 0 & 0 \\
\cline { 2 - 5 } & MAGOUMI & 0 & 0 & 0 \\
\cline { 2 - 5 } & OUEDEME & 0 & 0 & 0 \\
\cline { 2 - 5 } & THIO & 03,5 & 100 & 0 \\
\cline { 2 - 5 } & ZAFFE & 0 & 0 & 0 \\
\cline { 2 - 5 } & Sous-moyenne & $\mathbf{0 3 , 5}$ & $\mathbf{1 0 0}$ & $\mathbf{0}$ \\
\hline
\end{tabular}

Source : Enquêtes de terrain, juillet 2013

L'analyse du tableau VI montre que les superficies louées se retrouvent uniquement dans l'Arrondissement de Thio $(03,5 \mathrm{ha})$ et exclusivement à Glazoué. Seuls les hommes louent des terres à travailler. Les femmes sont donc marginalisées du fait de leurs conditions sociales qui les obligent à ne pas enfreindre les us et coutumes au point de subir des critiques acerbes, et de leur précarité économique. Selon les enquêtes de terrain, le coût du fermage d'une Kanti(Eso)qui est de $600 \mathrm{~m} 2 \mathrm{a}$ énormément évolué dans le secteur d'étude :25\% des enquêtés ont affirmé que ce coût de location par hectare avoisinait 1000 FCFA en 1992 et 3000 FCFA en 2002 ; alors qu'en 2012 il est de 50000 FCFA. Ce coût de location a été pratiquement multiplié par 50 en l'espace de 20 ans. Ce qui peut expliquer ce faible taux d'exploitants agricoles qui s'aventurent sur ce terrain.

Dans les Communes de Dassa-Zoumè et de Glazoué, on enregistre égalementle don comme mode d'accès à la terre.

\subsection{6-Don}

L'accès à la terre à Dassa-Zoumè et à Glazoué peut se faire aussi par "don" reçu par récompense de son détenteur après un loyal service rendu. Le tableau VII suivant donne une idée des superficies moyennes des terres acquises par Don et par sexe dans ces deux (02) communes.

Tableau VII : Superficie moyenne des terres acquises par 'don' et par sexe

\begin{tabular}{|c|c|c|c|c|}
\hline \multirow{4}{*}{ Communes } & Arrondissements & $\begin{array}{c}\text { Superficie } \\
\text { moyenne des } \\
\text { terres en don } \\
\text { (ha) }\end{array}$ & $\begin{array}{c}\text { Part des hommes } \\
\mathbf{( \% )}\end{array}$ & $\begin{array}{c}\text { Part des } \\
\text { femmes (\%) }\end{array}$ \\
\hline \multirow{4}{*}{$\begin{array}{c}\text { Dassa- } \\
\text { Zoumè }\end{array}$} & KERE & 02 & 100 & 0 \\
\cline { 2 - 5 } & KPINGNI & 0 & 0 & 0 \\
\cline { 2 - 5 } & PAOUINGNAN & 09 & 100 & 0 \\
\cline { 2 - 5 } & SOCLOGBO & 0 & 0 & 0 \\
\cline { 2 - 5 } & DASSA II & 0 & 0 & 0 \\
\hline \multirow{4}{*}{ Glazoué } & AKLANKPA & 0 & 0 & 0 \\
\cline { 2 - 5 } & MAGOUMI & 0 & 0 & 0 \\
\cline { 2 - 5 } & OUEDEME & 03 & 100 & 0 \\
\cline { 2 - 5 } & THIO & 0 & 0 & 100 \\
\cline { 2 - 5 } & ZAFFE & 07 & 0 & 0 \\
\hline
\end{tabular}




\begin{tabular}{|c|c|c|c|}
\hline Sous-moyenne & $\mathbf{0 5}$ & $\mathbf{3 0}$ & $\mathbf{7 0}$ \\
\hline Total & $\mathbf{1 0 , 5}$ & $\mathbf{6 7}$ & $\mathbf{3 3}$ \\
\hline
\end{tabular}

Source : Enquêtes de terrain, juillet 2013

D'après l'analyse du tableau VII, il ressort que les superficies données au niveau de ces deux (02) communes sont respectivementégales à 5,5 ha à Dassa-Zoumè et 5 ha à Glazoué. Toutefois, elles sont inégalement réparties entre les différents arrondissements où l'on observe que seulement deux (02) arrondissements sont concernés par cette répartition avec 2 ha et 9 ha respectivement à Kéré et à Paouignan dans la Commune de Dassa-Zoumè et 3 ha et 7 ha respectivement à Ouédémé et à Zaffé dans la Commune de Glazoué.

Quant à la part qui revient aux deux (02) sexes,il est à noter que seuls les hommes ont acquis des terres en 'don' à Dassa-Zoumèalors qu'à Glazoué, les deux (02) sexes ont acquis des terres dont $67 \%$ des hommes et $33 \%$ des femmes. Cette acquisition des deux sexes s'observe dans deux (02) arrondissements (Ouédèmé et Zaffé) à Glazoué. Ce mode d'accès à la terre est plus sécurisant par rapport à la location pour les propriétaires et constitue un atout pour la sécurité alimentaire. L'accès à la terre ne se fait pas que par le biais du don. Elle peut être également la propriété des collectivités.

\subsection{7- L'appropriation collective}

L'appropriation collective, l'un des modes d'acquisition de la terre à Dassa-Zoumè et à Glazoué, est détenue par 02,6\% de l'échantillon d'étude (tableau VIII). Cette propriété collective appartient à des familles notamment Doumatez, Kouloni, Zomahoun, et Koubiya à Dassa-Zoumè. Certaines de ces collectivités disposent encore de vastes domaines.

Tableau VIII :Superficie moyenne des terres en propriété collective

\begin{tabular}{|c|c|c|c|c|}
\hline \multirow{4}{*}{ Communes } & Arrondissements & $\begin{array}{c}\text { Superficie moyenne } \\
\text { des terres en } \\
\text { propriétés collectives } \\
\text { (ha) }\end{array}$ & $\begin{array}{c}\text { Part des hommes } \\
(\mathbf{\%})\end{array}$ & $\begin{array}{c}\text { Part des femmes } \\
(\%)\end{array}$ \\
\hline \multirow{5}{*}{ Dassa-Zoumè } & KERE & 02 & 100 & 0 \\
\cline { 2 - 5 } & KPINGNI & 02 & 100 & 0 \\
\cline { 2 - 5 } & PAOUINGNAN & 02 & 100 & 0 \\
\cline { 2 - 5 } & SOCLOGBO & 0 & 0 & 0 \\
\cline { 2 - 5 } & DASSA II & 0 & 0 & 0 \\
\hline \multirow{5}{*}{ Glazoué } & Sous-moyenne & $\mathbf{0 2}$ & 0 & 0 \\
\cline { 2 - 5 } & AKLANKPA & 0 & 0 & 0 \\
\cline { 2 - 5 } & OUGOUMI & 0 & 0 & 0 \\
\cline { 2 - 5 } & OUEDEME & 0 & 0 & 0 \\
\cline { 2 - 5 } & THIO & 0 & 0 & 0 \\
\cline { 2 - 5 } & Sous-moyenne & 0 & 0 & 0 \\
\hline
\end{tabular}


Source : Enquêtes de terrain, juillet 2013

L'analyse de la superficie moyenne des terres en propriété collective montre que trois (03) arrondissements (Kéré, Kpingni, Paouingnan) sur cinq (05) ont le même nombre d'hectares de superficies avec une moyenne de 02 ha à Dassa-Zoumè alors qu'à Glazoué, ces propriétés collectives ont disparu. Donc, seul Dassa-Zoumè dispose encore de terres appartenant aux collectivités.L'absence de propriétés collectives à Glazoué peut s'expliquer par l'émiettement de ces terres et l'individualisme qui imprime aujourd'hui sa marque dans presque toutes nos contrées. En effet, cet émiettement des terres et l'individualisme sont le reflet des tensions sociales qui résultent du droit de cité sur la terre au sein d'une même collectivité et les problèmes économiques de plus en plus récurrents de nos jours font que chaque individu cherche à avoir sa propre parcelle pour mieux la contrôler.

\section{2- Caractérisation des pratiques agricoles}

Dans les Communes de Dassa-Zoumè et de Glazoué, la durée moyenne d'utilisation de la terre et la durée de jachère varient d'un arrondissement à un autre (tableau IX).

Tableau IX : Durées de mise en culture et de la jachère à Dassa-Zoumè et à Glazoué

\begin{tabular}{|c|c|c|c|}
\hline Communes & Arrondissements & $\begin{array}{l}\text { Durée de mise en } \\
\text { culture (année) }\end{array}$ & $\begin{array}{l}\text { Durée de jachère } \\
\text { (année) }\end{array}$ \\
\hline \multirow{7}{*}{ Dassa-Zoumè } & KERE & 11 & 10 \\
\hline & KPINGNI & 05 & 07 \\
\hline & PAOUINGNAN & 12 & 06 \\
\hline & SOCLOGBO & 05 & 05 \\
\hline & DASSA II & 05 & 05 \\
\hline & Sous-moyenne & 08 & 07 \\
\hline & Coefficient de Ruthenberg & \multicolumn{2}{|c|}{53,33} \\
\hline \multirow{6}{*}{ Glazoué } & AKLANKPA & 05 & 06 \\
\hline & MAGOUMI & 14 & 05 \\
\hline & OUEDEME & 13 & 04 \\
\hline & THIO & 04 & 05 \\
\hline & ZAFFE & 08 & 05 \\
\hline & Sous-moyenne & 09 & 05 \\
\hline \multicolumn{2}{|c|}{ Coefficient de Ruthenberg } & \multicolumn{2}{|c|}{64,28} \\
\hline & Moyenne & 08 & 06 \\
\hline \multicolumn{2}{|c|}{ Coefficient de Ruthenbergde l'ensemble } & \multicolumn{2}{|c|}{57,14} \\
\hline
\end{tabular}

Source :Résultats d'enquêtes de terrain, juillet 2013

L'analyse du tableau IX montre que les moyennes des durées de mise en culture et de jachère à Dassa-Zoumè sont sensiblement les mêmes (respectivement 08 ans et 07 ans). Par contre à Glazoué, la moyenne de la 
durée de mise en culture est plus longue (09 ans) que celle de la durée de la jachère (05 ans). Ainsi, au niveau des deux (02) communes, la moyenne de la durée de mise en culture est de 08 ans alors que celle de la durée de la jachère est de 06 ans.

Le coefficient de Ruthenberg calculé est compris entre $33<\mathrm{R}<66$, soit $\mathrm{R}=57,14$ etpermet d'affirmer que c'est le système de jachère qui est le plus adopté par les exploitants agricoles dans les deux (02) communes. Les résultats issus des enquêtes de terrain le confirment. En effet, sur l'ensemble de l'effectif des enquêtés, plus de $79 \%$ ont affirmé avoir des terres en jachère du fait de leur pauvreté et des conflits fonciers. Mais, les systèmes culturaux appliqués par les exploitants agricoles varient selon les moyens, les types de sols et la disponibilité en main-d'œuvre et en terres cultivables. La figure 2 montre les différentes catégories d'exploitants agricoles et les superficies des terres cultivables dans les deux (02) communes.

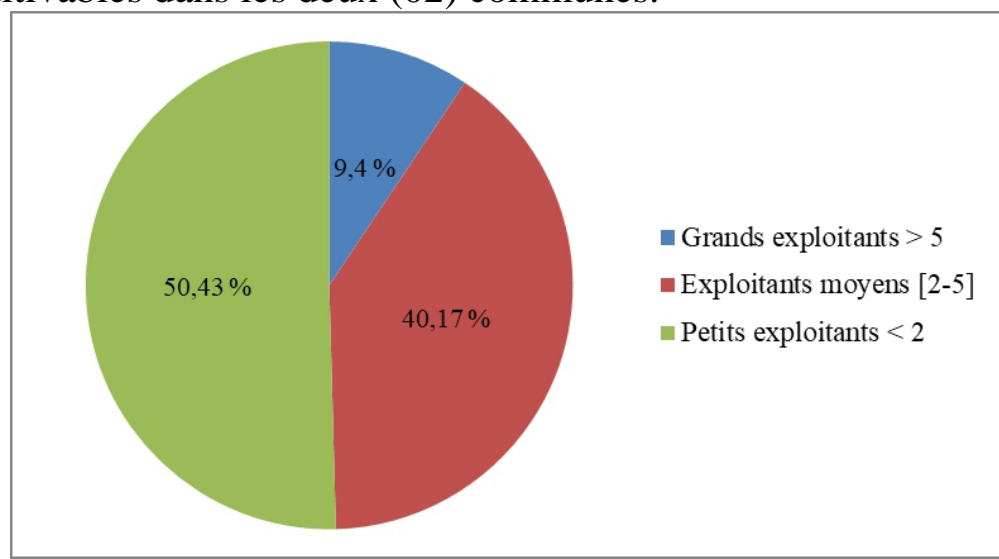

Figure 2 : Catégories d'exploitants agricoles suivant les superficies des terres cultivables Source : Résultats d'enquête de terrain, juillet 2015

La figure 2montre que les superficies des terres cultivables sont de plus en plus fractionnées par les exploitants agricoles et que les petits exploitants se retrouvent avec moins de deux (02) hectaressoit 50,43\%. Les grands exploitants ( $>5 \mathrm{ha}$ ) occupent seulement 09,40\% de l'échantillon d'enquête, preuve que l'individualisme commence à gagner les exploitants agricoles. Cet individualismepermet d'avoir seulement une production de subsistance, et peut être à l'origine de l'incapacité des exploitants agricoles à constituer des stocks pour faire face aux périodes de soudure, donc à l'insécurité alimentaire. Les exploitants moyens [2-5] occupent 40,17 \% de l'échantillon d'étude. En dehors de la jachère, il existe également d'autres techniques culturales utilisées par les exploitants agricoles.Ainsi, certains de ces exploitants agricoles pour remédier à cet état de choses ne manque pas d'imagination. Ils y associent d'autres activités génératrices de revenus comme l'illustre les photos 1 a et $b$. 


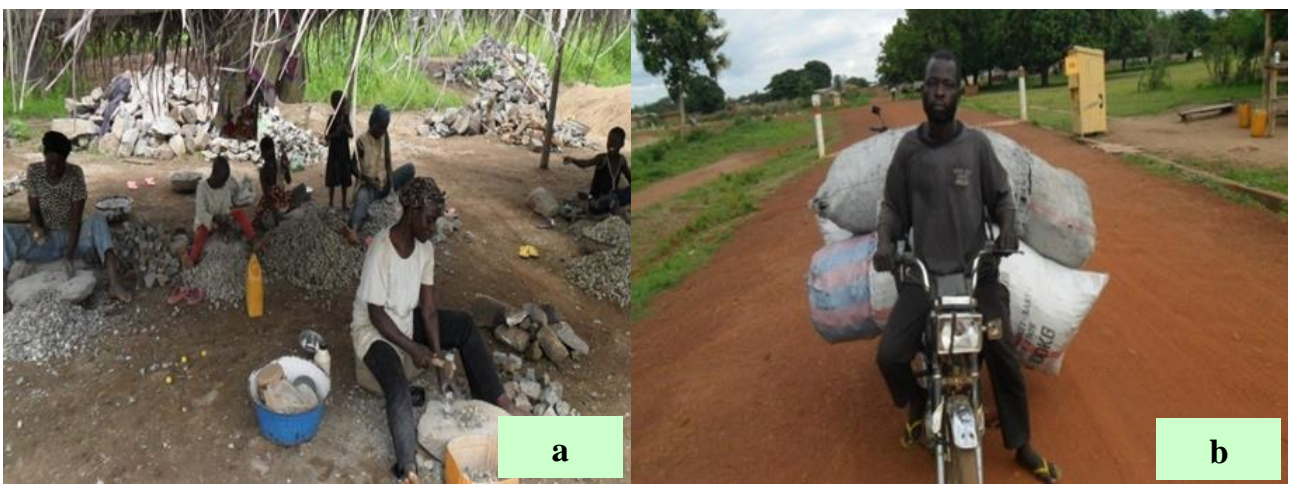

Photo 1: Concassage de granites à Dassa II (Dassa-Zoumè)
Photo 2 : Transport du charbon de bois à Thio (Glazoué)

Les photos 1 et 2 montrent quelques-unes des activités économiques pratiquées par les exploitants agricoles pour passer avec moins de difficultés possibles les périodes de soudure. En 1, on peut observer des activités de concassage de granites exercées par les exploitant (e)s agricoles (femmeset enfants) à Dassa-Zoumè. En b, il s'agit d'un exploitant agricole qui, après transformation des bois en charbon de bois, les converge vers le marché pour la vente.

\section{Discussions}

Les différents modes d'accès à la terre enregistrés dans les Communes de Dassa-Zoumè et de Glazoué montrent l'existence de sept (07) modes d'accès à la terre : l'héritage, l'achat, l'emprunt, le métayage, le fermage ou location, le "don", et l'appropriation collective. Les résultats issus des modes d'accès à la terre dans ces deux (02) communes sont presque conformes à ceux trouvés par Loconon (1987), Biaou (1991), Honlonkou (1999), Fangnon (2012) qui ont recensé les mêmes modes d'accès à la terre sauf que le "don"' a disparu dans leurs milieux d'étude respectifs.

Le métayage pratiqué dans les deux (02) communes d'étude ne permet en aucun cas au métayer de devenir propriétaire de la terre. Donc, ce résultat trouvé est contraire à celui de Biaou (1991) selon lequel le métayage encore appelé "dimi-madimi" pratiqué au Togo permet au tenancier (métayer), après un long moment, de devenir propriétaire de la terre, la plantation étant subdivisée en deux (02) parties, moitié pour lui et moitié au propriétaire foncier.

A Klouékanmè, Azontondé (2004) trouve que le mode de faire-valoir dominant est la location $(35,83 \%)$. Ce fort taux de location est dû à la mauvaise répartition des terres et au fait que les femmes n'ont pas droit à l'héritage des terres. Biaou (1985) fait remarquer que les différents modes d'occupation de la terre identifiés par les chercheurs sont entre autres l'achat, 
l'héritage, la location, le métayage, le prêt, l'attribution à titre gratuit. L'augmentation sans cesse de l'effectif de la population est à la base de diversité des stratégies d'obtention de la ressource précieuse qu'est la terre.

Dans le même ordre d'idées, Platteau (1993) affirme que «l'accès à la terre n'est pas seulement fondé sur des liens de parenté ou sur la descendance, mais aussi sur des rapports moraux ou de protection qui sont souvent associés à certaines formes acquises de statut ou d'identité sociale». Cela signifie pour Biaou (1993) que les lignages ou les groupes sociaux ayant un excédent de superficies cultivables peuvent toujours accepter des étrangers en quête de terre à condition que ceux-ci acceptent de s'intégrer au système local de relations réciproques et reconnaissent le pouvoir du chef local.

Sanou (2004) trouve pour sa part que chez les Bobo Sya Da au Burkina Faso, le mode encore en vigueur est le don. La demande est adressée au chef de village ou au chef de lignage. A la fin des récoltes, le bénéficiaire de la terre (l'étranger) doit donner à son hôte un panier de mil symbolique. Biaou (1991) fait ressortir qu'avec l'évolution de la vie socio-économique, d'autres formes d'acquisition de terrain fondées essentiellement sur des contrats de donation, de prêt, de mise en gage, de location, de métayage font leur apparition et font légion dans certaines régions comme le plateau Adja par exemple. Pour Adou Yao (2004), dans la forêt classée de Monogaga en Côte d'Ivoire, la terre est transmise de génération en génération selon les logiques lignagères.

Les étrangers y accèdent à travers des contrats. Ces milieux ne subissent pas encore la contrainte foncière. Mais malgré cela, Biaou (1993) note que l'émergence et le développement du marché de la terre ont fini par faire du principe d'achat de terre un élément prédominant dans les modes d'acquisition après les héritages. En effet, l'achat, l'héritage et le don accordent plus de liberté. L'exploitant peut faire sans contrainte sur ces terres les cu1tures qu'il veut. Quant à la location et le métayage ils accordent moins de liberté. Le locataire se trouve dans l'obligation de payer la rente. Son objectif serait alors de rentabiliser financièrement cette terre. Les rapports fonciers dans 1esquels sont entrées des sommes d'argent tendent à privilégier des produits agricoles économiquement rentables sur le marché en terme de prix de vente.

Par ai1leurs, Fangnon (2012) révèle que le système de 1ocation inconnu traditionne1lement qui, actuellement, s'est développé, répandu et renforcé, n'est qu'une des conséquences de l'entrée de la terre dans les rapports de marché c'est-à-dire dans une économie devenue de plus en plus monétaire. Le métayer dans 1e besoin de terres cultivables et devant une insuffisance d'argent pour en acheter ou en louer, se trouve obligé de conclure des contrats avec les propriétaires terriens sur 1a base du partage des produits. Généralement, 1es propriétaires exigent la mise en place des cultures de subsistance en l'occurrence le maïs sur ces parcelles. Dès lors, 1a liberté du 
choix des cultures sera relativement plus grande si la volonté du paysan n'est pas conditionnée par la nature du contrat foncier avec le propriétaire.

En effet, selon Faure (1990), la structure du régime foncier, régi par le droit coutumier, empêche les jeunes exploitants dynamiques de mobiliser la main-d'œuvre et les moyens matériels comme ils le souhaiteraient. Ces chercheurs ont également souligné l'allocation non efficience des ressources sous le régime du métayage ; conclusion soutenue par Cheung (1969) sur la théorie marshallienne.

\section{Conclusion}

L'étude des influences des modes d'accès à la terresur la production agricole dans les Communes de Dassa-Zoumé et de Glazoué a montré l'existence de 07 modes d'accès à la terre (l'héritage, l'achat, l'emprunt, le métayage, le fermage (location), le "don' et l'appropriation collective) qui constituent soit des atouts ou des contraintes à la production agricole.

\section{References:}

1. ADOU, Y.C., (2004) : Actions de la Sodefor et pratiques paysannes des Wanne dans la forêt classée de Monogaga (Côte d'Ivoire), In Annales de l'Université Abdou Moumouni, nspécial, pp 173-186.

2. AFOUDA, F., (1990) : L'eau et les cultures dans le Bénin central et septentrional : étude de la variabilité des bilans de l'eau dans leurs relations avec le milieu rural de la savane africaine, Thèse de doctorat nouveau régime, Paris IV, Sorbonne, $428 \mathrm{p}$.

3. AGOSSOU, V.A.M., et IGUE, J., (2002) : Caractérisation paysanne et scientifique des sols des sites d'expérimentation agricole de la région centre du Bénin, In Actes de l'Atelier Scientifique Centre 1, pp. 136150 .

4. AZONTONDE, R., (2004) : Impact économique de l'adoption des pratiques de la Gestion Intégrée de la Fertilité des Sols (GIFS) au sudBénin : cas d'Ahohoué (Commune de Klouékanmè) et de Banigbé (Commune d'Ifangni), Thèse pour l'obtention du diplôme d'ingénieur agronome, FSA/UAC, $154 \mathrm{p}$.

5. BADOUIN, R., (1985): Le développement agricole en Afrique tropicale, Cujus, Katharla, Paris, pp 18-24.

6. BIAOU, G., (1985): Etude de quelques exploitations agricoles paysannes dans le district rural de Klouékanmè. Thèse pour l'obtention du diplôme d'Ingénieur agronome, FSA, 192 p.

7. BIAOU, G., (1991) : Régime foncier et gestion des exploitations agricoles sur le plateau Adja, Bénin. Thèse de Doctorat de $3^{\text {è }}$ cycle en Economie Rurale. Côte d'Ivoire: CIRES (Centre Ivoirien de Recherches Economiques et Sociales), 207 p. 
8. BIAOU, G.,(1993):Le développement du marché de la terre sur le Plateau Adja ; inSystèmes agricoles en Afrique, Volume 3, $\mathrm{n}^{\circ} 2$ : pp 54-63

9. BOKO, M., (1988) : Climats et communautés rurales du Bénin : rythmes climatiques et rythmes de développement. Thèse d'Etat. 2 tomes. Centre de recherches en Climatologie. URA 909 CNRS, Université de Bourgogne, Dijon, 601 p.

10. BOKO, M.,(1998) :Climat, eau et société au Bénin.In Annales de la FLASH, $\mathrm{N}^{\circ} 6$, pp 53-59.

11. BOKO, M., (2004) : Agriculture durable et gestion des ressources naturelles, CIFRED/UAC, pp 110-124.

12. CHAUVEAU, J.P., COLIN, J.P., JACOB, J.B., DELVILLE, P.I.., LE MEUR, E., (2006) :Modes d'accèsà laterre, marchés fonciers, gouvernance et politiques foncières en Afrique de l'Ouest : résultats du projet de recherche CLAIMS. Londres: IIED, 92 p.

13. CHEUNG, S.N.S., (1969): The theory of share tenancy, The University of Chicago Press, London, $89 \mathrm{p}$.

14. DE SCHUTER, O., (2010): Access to land and the right to food', Report of the Special Reporter on the right to food presented at the 65 the General Assembly of United Nations (A/65/281),22p.(Enligne)URL:htpp://www.srfood.org/images/stories/ pdf/officialreports/20101021_access-to-land-report_en pdf (consulté le 12/10/2017).

15. DJAOUGA, M., (2006) : Dynamique des écosystèmes agropastoraux périurbains dans le Borgou : Cas de la ville de Parakou. Mémoire de DEA en Géographie, EDP /FLASH/UAC, 85p.

16. ERPICUM, R., (2004) : Discours d'accueil du Colloque Scientifique de N'Djamena sur la question foncière au Tchad, 28 juin au $1^{\text {er }}$ juillet 2004 in Actes du Colloque, CEFOD-OFT, Septembre 2004, p 10.

17. FANGNON, B., (2012) : Qualité des sols, systèmes de production agricole et impacts environnementaux et socio-économiques dans le Département du Couffo au sud-ouest du Bénin,Thèse de Doctorat de Géographie, EDP/FLASH/UAC, 308 p.

18. FAURE, A., (1990) :L'appropriation de l'espace foncier : une étude d'anthropologie sociale en région Bissa Burkina Faso; Thèse de doctorat de sociologie. EHESS, Paris, 456 p.

19. HONLONKOU, N.A., (1999) : Impact économique des techniques de fertilisation des sols : cas de la jachère Mucuna au sud du Bénin, Thèse de Doctorat de $3^{\text {è }}$ cycle, CIRES, Côte d'ivoire, 187 p.

20. HOUEDJISSIN, C.R., (2000) : Impacts environnementaux des modes de communalité et de gestion des terres dans la Sous-Préfecture 
d'Agbangnizoun, Mémoire de DEA de Géographie, EDP/FLASH/UNB, $91 \mathrm{p}$.

21. INSAE, (2013) : Quatrième Recensement Général de la Population et de l'Habitation (RGPH 4), 87 p.

22. LOCONON, Z.D., (1987): Analyse socio-économique de quelques systèmes d'exploitation agricole en vue de l'élaboration d'une typologie sur le plateau Adja (Province du Mono). Etude de cas de trois villages, Thèse d'Ingénieur agronome, FSA, $131 \mathrm{p}$.

23. ODJO, P., (1997) : Potentialités agricoles et sécurité alimentaire dans la Commune de Tchaourou, Mémoire de maîtrise de Géographie, DGAT/ FLASH/UAC, $64 \mathrm{p}$.

24. PLATTEAU, J., (1993) : « L'influence des politiques foncières coloniales ». Études FAO : 107, pp 105-121.

25. SANOU, A., (2004) : La gestion de la terre et la représentation rituelle chez les Bobo Sya Da (Burkina Faso), In Annales de l'Université Abdou Moumouni, ${ }^{\circ}{ }^{\circ}$ spécial, pp 187-193.

26. SCHWARTZ，D., (1995):Méthodes statistiques à l'usage des médecins et des biologistes. $4^{\mathrm{e}}$ édition (Editions médicales Flammarion), Paris, $314 \mathrm{p}$.

27. TENTE, B.; SINSIN, B., (2002) : Diversité et structure des formations arborescentes du secteur Perma-Toucountouna dans la chaîne de l'Atacora (Bénin). Vég. Burkina Faso 6 : pp 31-42.

28. WARTENA, D., (1987): Le système économique des Adja en développement : attitudes vis-à-vis des changements dans les villages Atindéhouhoué et Housouhoué du district de Toviklin, Province du Mono, Bénin. Projet UNB/LUW/SVR, Abomey-Calavi, 303 p.

29. WOKOU, G.C., (2009) : Agriculture et environnement sur le plateau d'Agonlin : systèmes culturaux et mutations environnementales, Mémoire de DEA de Géographie, EDP/FLASH/UAC, 92 p. 